\title{
Variable association of height with gastric cancer by anatomical subsite
}

\author{
M. C. Camargo' ${ }^{1}$ C. S. Rabkin ${ }^{1}$ N. Appel ${ }^{2}$ A. R. Hollenbeck ${ }^{3}$
}

Received: 5 January 2015 / Accepted: 9 June 2015/Published online: 20 June 2015

(C) Springer International Publishing Switzerland (outside the USA) 2015

To the Editor. Dr. Kabat and colleagues [1] presented an analysis of attained height with risk of cancer at different anatomical sites in a US cohort and reported no association in either sex for gastric cancer overall. It is well known that cancers of the gastric cardia and noncardia subsites have distinct etiological factors. In particular, seropositivity for Helicobacter pylori is positively associated with noncardia gastric cancer but inversely associated with cardia cancer. For this reason, in our previous study of nearly the same cohort (restricted to whites) [2], we evaluated associations of height tertiles with gastric cancer separately for each anatomical subsite. We found that noncardia gastric cancer was inversely associated with height and interpreted the excess risk for shorter adults to be consistent with the known association of chronic H. pylori infection with growth retardation during childhood [3]. Nevertheless, it cannot be completely dismissed that $H$. pylori infection may be a surrogate marker for other factors associated with socioeconomic deprivation in early life. We did not find an association of height with cardia cancer.

Using the same population (all races), covariable adjustments and height metric as described by Dr. Kabat and colleagues, we have evaluated subsite-specific associations of gastric cancer risk with a $10-\mathrm{cm}$ increase in height. Consistent with our previous findings, noncardia

M. C. Camargo

camargomc@mail.nih.gov

1 Division of Cancer Epidemiology and Genetics, National Cancer Institute, 9609 Medical Center Dr., Rm. 6E-110, Bethesda, MD 20892, USA

2 Information Management Services, Inc., 6110 Executive Blvd, Suite 310, Rockville, MD 20852, USA

3 AARP, 601 E St NW, Washington, DC 20049, USA gastric cancer was inversely associated with height in both sexes combined, with a hazard ratio (HR) of 0.84 per $10-\mathrm{cm}$ increase (95\% confidence interval [CI] 0.72-0.98), and no evidence for effect modification by sex (HRs of 0.80 in men and 0.94 in women; $p$-interaction $=0.5$ ). Height was not associated with gastric cardia cancer in this alternative approach (HR 1.11; $95 \%$ CI 0.98-1.25).

In conclusion, the association of height with gastric cancer in this cohort varies by anatomical subsite, suggesting the need to consider potential etiological heterogeneity in studies of cancer risk.

\section{References}

1. Kabat GC, Kim MY, Hollenbeck AR, Rohan TE (2014) Attained height, sex, and risk of cancer at different anatomic sites in the NIH-AARP diet and health study. Cancer Causes Control 25:1697-1706

2. Camargo MC, Freedman ND, Hollenbeck AR, Abnet CC, Rabkin CS (2014) Height, weight, and body mass index associations with gastric cancer subsites. Gastric Cancer 17:463-468

3. Goodman KJ, Correa P, Mera R, Yepez MC, Cerón C et al (2001) Effect of Helicobacter pylori infection on growth velocity of school-age Andean children. Epidemiology 22:118-126 